\title{
Study on a Cracked Single-Layer 2D Woven Composite Plate: Fracture Mode and Damage Analysis
}

\author{
Y. Q. Wang, ${ }^{a}$ P. Cheng, ${ }^{a}$ X. S. Liu, ${ }^{\text {b }}$ Y. J. Bao, ${ }^{\text {a,1 }}$ and H. Gao ${ }^{a}$ \\ ${ }^{a}$ Key Laboratory for Precision and Nontraditional Machining Technology of Ministry of Education, \\ School of Mechanical Engineering, Dalian University of Technology, Dalian, China \\ ${ }^{\mathrm{b}}$ School of Automotive Engineering, Dalian University of Technology, Dalian, China \\ ${ }^{1}$ byj@dlut.edu.cn
}

An experimental study and numerical calculation are conducted to investigate the crack propagation in a $2 D$ woven composite. Crack propagation tests of single-layer composite plate specimens permit this process to be directly followed. Analysis of their fracture surfaces reveals crack propagation paths, fracture mode, and damage mechanisms. The load-deflection curve represents the crack propagation as a three-stage process. The stress intensity near the crack tip is evaluated with the critical stress intensity factor numerically calculated. The results demonstrate a good agreement between the calculations and experiment.

Keywords: woven carbon fibers, crack propagation, fracture mode, damage, stress intensity factor.

Introduction. Woven composites outperform most conventional materials by lightweight, strength, and stiffness characteristics, as well as better structural design performance. Therefore, they are widely utilized as the bearing structure components in aerospace and other high-tech fields. However, woven composites are prone to the formation of holes, cracks, delamination, etc. These factors produce high stress intensity factors, increase the damage, and enhance fracture [1]. So far, the crack propagation of a unidirectional composite has been studied extensively both in terms of fracture mode and fatigue crack propagation [2, 3]. Czabaj and Ratcliffe [4] has studied the fracture-toughness of a unidirectional graphite/epoxy composite under Mode-I loading. Arguelles et al. [5] have investigated the Mode-I crack initiation and growth rate of a carbon-fiber epoxy composite under fatigue loading. Donadon et al. [6] and Blanco et al. [7] also have explored the fracture behavior of woven fiber laminates. However, they examined the cracking of multi-layer laminates, without a direct observation of the crack propagation process. At present, only a few scholars studied the cracking of uni-layer composite plates, most of which focused on the buckling of pre-cracked plates under compression [8].

In this work, specimens of uni-layer laminates manufactured through a method of RTM have been used in all tests. According to the crack propagation test of the uni-layer laminates, the crack propagation can be observed directly. The aim of this study is to analyze cracked CFRP specimens, with crack propagation by Mode I. The stress intensity factors (SIF) at the crack tip are calculated, and trends of crack propagation are explored.

1. Extended Finite Element Modeling. The extended finite element method (XFEM) is widely used in fracture analysis, especially for the problems of local discontinuity and singularity. Now, development of XFEM has substantially contributed to crack propagation analysis of various types of composite materials [9]. Due to the anisotropy of the composite material, anisotropic enrichment functions are embedded into an anisotropic finite element procedure. The extended finite element method is be able to solve the problem of anisotropy. By using the generalized Heaviside and crack-tip (near tip) function, several degrees of freedom (DoF) are added for the nodes near discontinuities [10]. 
1.1. The Enriched Approximation for the Displacement Field. The approximation of displacement for point $X$ located within the domain can be described by the XFEM approach as follows $[10,11]$ :

$$
\begin{gathered}
u^{h}(X)=\sum_{\substack{I \\
n_{I} \in k^{2}}} \phi_{I}(X) u_{I}+\sum_{\substack{J \\
n_{J} \in N^{g}}} b_{J} \phi(X) H(X)+\sum_{k=K^{1}} \phi_{k}(X)\left(\sum_{l} c_{k}^{l 1} F_{l}^{1}(X)\right)+ \\
+\sum_{k=K^{2}} \phi_{k}(X)\left(\sum_{l} c_{k}^{l 2} F_{l}^{2}(X)\right)
\end{gathered}
$$

where $b_{J}$ is the vector of additional degrees of nodal freedom, $N^{g}$ is the set of nodes that have a crack face in their support domain, $c_{k}$ is the nodal freedom of the crack-tips of the vector, $F_{l}^{i}(X)(i=1,2)$, are crack-tip enrichment functions, and $H(X)$ is the generalized Heaviside function [11], it is simulated the displacement discontinuity of the crack faces. If $X$ is above the crack, $H(X)$ has value +1 and -1 values, otherwise. If $X^{*}$ is the nearest point on the crack to $X$ and $e_{n}$ is the unit vector normal to the crack alignment, then

$$
H(X)= \begin{cases}1 & \text { if }\left(X-X^{*}\right) e_{n} \geq 0 \\ -1 & \text { otherwise }\end{cases}
$$

1.2. Crack-Tip Functions. The crack-tip enrichment $F_{l}$ values are extracted in a way to include all possible displacement states. According to $[9,12,13]$, the crack-tip functions can be defined as

$\left\{F_{l}(r, \theta)\right\}_{l=1}^{4}=\left\{\sqrt{r} \cos \frac{\theta_{1}}{2} \sqrt{g_{1}(\theta)}, \sqrt{r} \cos \frac{\theta_{2}}{2} \sqrt{g_{2}(\theta)}, \sqrt{r} \sin \frac{\theta_{1}}{2} \sqrt{g_{1}(\theta)}, \sqrt{r} \sin \frac{\theta_{2}}{2} \sqrt{g_{2}(\theta)}\right\}$,

where functions $g_{k}(\theta)$ and $\theta_{k}$, in their general form for all orthotropic composites, are defined as

$$
\begin{gathered}
g_{k}(\theta)=\sqrt{\left(\cos \theta+s_{k x} \sin \theta\right)^{2}+\left(s_{k y} \sin \theta\right)^{2}}, \\
\theta_{k}=\arctan \left(\frac{s_{k y} \sin \theta}{\cos \theta+s_{k y} \sin \theta}\right) .
\end{gathered}
$$

When $a_{1}>\sqrt{a_{2}}$ (type I),

$$
\begin{gathered}
g_{j}(\theta)=\left(\cos ^{2} \theta+\frac{\sin ^{2} \theta}{e_{j}^{2}}\right)^{1 / 2}, \quad j=1,2, \\
\theta_{j}=\tan ^{-1}\left(\frac{x_{2}}{e_{j} x_{1}}\right)=\tan ^{-1}\left(\frac{\tan \theta}{e_{j}}\right), \quad j=1,2,
\end{gathered}
$$

where $e_{1}$ and $e_{2}$ are defined as $[14,15]$ : 
$e_{1}=\left\{\frac{1}{2}\left[\frac{d_{66}}{d_{11}}+\frac{d_{22}}{d_{66}}-\frac{\left(d_{12}+d_{66}\right)^{2}}{d_{11} d_{66}}\right]-\left(\frac{1}{4}\left[\frac{d_{66}}{d_{11}}+\frac{d_{22}}{d_{66}}-\frac{\left(d_{12}+d_{66}\right)^{2}}{d_{11} d_{66}}\right]-\frac{d_{22}}{d_{11}}\right)^{1 / 2}\right\}^{1 / 2}$,
$e_{2}=\left\{\frac{1}{2}\left[\frac{d_{66}}{d_{11}}+\frac{d_{22}}{d_{66}}-\frac{\left(d_{12}+d_{66}\right)^{2}}{d_{11} d_{66}}\right]+\left(\frac{1}{4}\left[\frac{d_{66}}{d_{11}}+\frac{d_{22}}{d_{66}}-\frac{\left(d_{12}+d_{66}\right)^{2}}{d_{11} d_{66}}\right]^{2}-\frac{d_{22}}{d_{11}}\right)^{1 / 2}\right\}^{1 / 2}$.

When $\left|a_{1}\right|<\sqrt{a_{2}}$ (type II), the functions are

$$
\begin{aligned}
& g_{j}(\theta)=\left(\cos ^{2} \theta+l^{2} \sin ^{2} \theta+(-1)^{j} l^{2} \sin 2 \theta\right)^{1 / 2}, \\
& \theta_{j}=\arctan \left(\frac{\gamma_{2} l^{2} \sin \theta}{\cos \theta+(-1)^{j} \gamma_{1} l^{2} \sin \theta}\right), \quad j=1,2,
\end{aligned}
$$

where $\gamma_{1}, \gamma_{2}$, and $l^{2}$ are defined as $[12,16]$ :

$$
\begin{gathered}
\gamma_{1}=\left[\frac{1}{2}+\left(\sqrt{a_{2}+a_{1}}\right)\right]^{1 / 2}, \\
\gamma_{2}=\left[\frac{1}{2}+\left(\sqrt{a_{2}-a_{1}}\right)\right]^{1 / 2}, \\
l^{2}=\left(\gamma_{1}^{2}+\gamma_{2}^{2}\right)^{-1} .
\end{gathered}
$$

\section{Material and Specimen Fabrication.}

2.1. Materials. A carbon fiber $2 \mathrm{D}$ woven reinforced epoxy resin matrix laminated composite was used for the tests. The composites are composed of T300 carbon fibers (TORAY, Japan) impregnated with toughened E51 epoxy resin (Wuxi Qian Guang Chemical Raw Materials Co. Ltd., China) by RTM method. The carbon fiber is woven into a plain pattern, and the schematic structure is presented in Fig. 1. The fiber and resin contents are 45 and $55 \%$ by volume, respectively. The thickness of the uni-layer composite plate is $0.2 \mathrm{~mm}$. The main mechanical properties of the plate are listed in Table 1 [17].

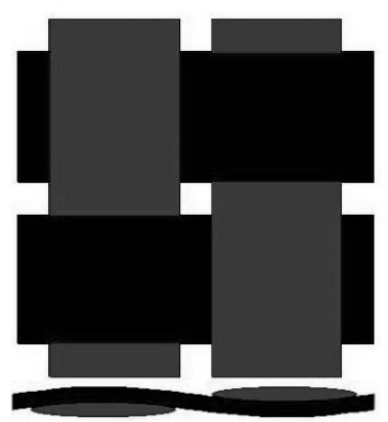

Fig. 1. The schematic structure of the plain woven pattern. 
$\mathrm{T}$ a $\mathrm{b} 1 \mathrm{e} 1$

The Mechanical Properties of the Woven Composite Material

\begin{tabular}{|c|c|c|c|c|c|c|c|c||}
\hline $\begin{array}{c}E_{1}, \\
\mathrm{GPa}\end{array}$ & $\begin{array}{c}E_{2}, \\
\mathrm{GPa}\end{array}$ & $\begin{array}{c}E_{3}, \\
\mathrm{GPa}\end{array}$ & $\begin{array}{c}G_{12}, \\
\mathrm{GPa}\end{array}$ & $\begin{array}{c}G_{13}, \\
\mathrm{GPa}\end{array}$ & $\begin{array}{c}G_{23}, \\
\mathrm{GPa}\end{array}$ & $v_{12}$ & $v_{13}$ & $v_{23}$ \\
\hline 26.7 & 26.7 & 9.36 & 2.4 & 2.4 & 2.64 & 0.13 & 0.36 & 0.36 \\
\hline
\end{tabular}

2.2. Parameters of Test Specimens and Pre-Cracking. According to study [18], the specimens are fabricated with the following dimensions: width of $40 \mathrm{~mm}$, thickness of $0.2 \mathrm{~mm}$, length of $90 \mathrm{~mm}$. The pre-crack lengths were $0,4,8,12,16,18$, and $20 \mathrm{~mm}$, respectively. Sandpaper protection tabs (20 mm-long and $0.2 \mathrm{~mm}$-thick) were placed on both edges of the specimen. The lower part of the specimen has a scale to facilitate the measurement of the crack propagation length as shown in Fig. 2. The specimens were cut via the wire cutting machine, according to the specified size along the fiber bundle gap, without touching the fiber bundle. The location of the pre-crack tip is shown in Fig. 3.

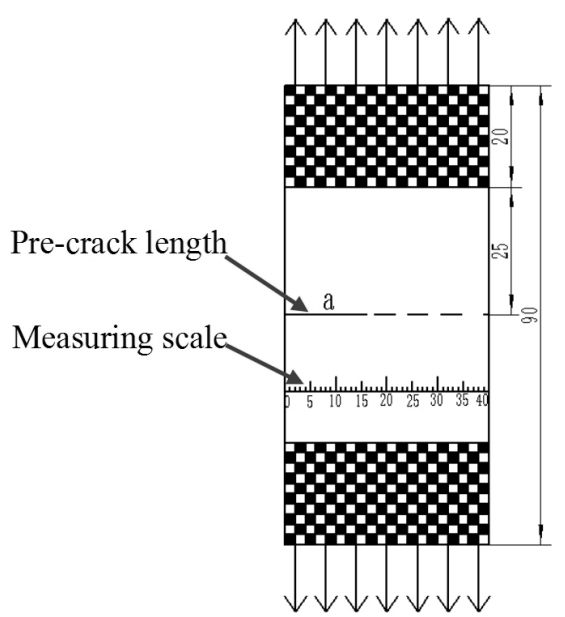

Fig. 2

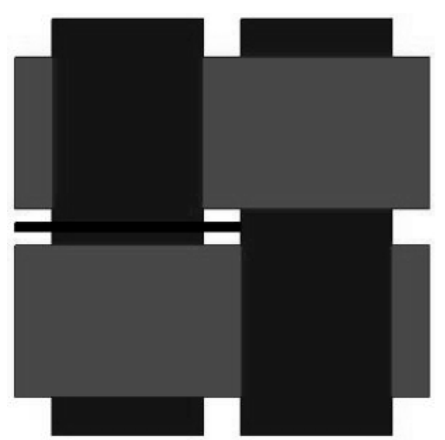

Fig. 3

Fig. 2. Size of experimental specimens and test loading method.

Fig. 3. The location of the pre-crack tip with plain woven CFRP.

3. Experimental Procedures. Mode I crack propagation tests were conducted on the universal testing machine (WDW-100) for specimens with different pre-crack lengths. The specimen dimensions are shown in Fig. 2, while the loading mode is direct tension in the vertical pre-crack direction. As the thickness is relatively low, the propagation speed is too fast. Therefore, the specimens were loaded by displacement control with a rate of $0.1 \mathrm{~mm} / \mathrm{min}$. When the loading was started, the camera recorded the whole process of crack propagation, as shown in Fig. 4.

\section{Results and Discussion.}

4.1. Damage Types and Crack Propagation Path. Since specimens under study were uni-layer composite plates, the crack propagation, fracture surfaces, and fracture mode could be observed directly. Observing the specimens after the experiment, the warp bundles perpendicular to the crack propagation direction, the two interweaving points between the weft bundles, and warp bundles were found to be high-SIF zones. As seen in Fig. 5, the expansion of the crack was easy to ensure in the direction parallel to the crack propagation direction with the fiber debonding, breaking, and debonding of the warp and weft fiber bundles, whereas the propagation process exhibited dynamic patterns. 


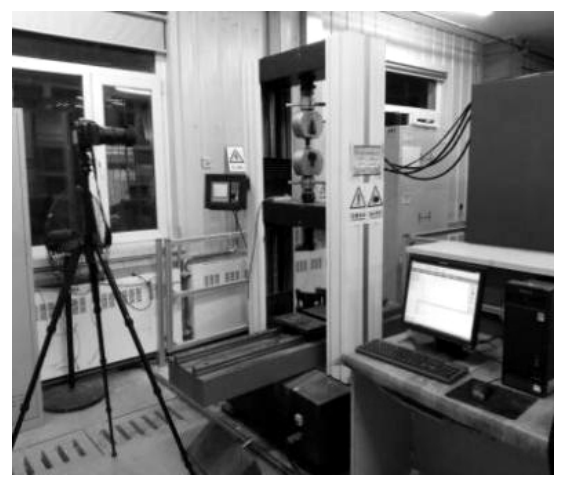

a

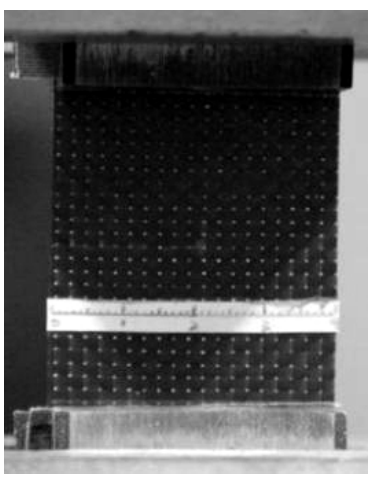

b

Fig. 4. Overall crack propagation experiment configuration (a) and clamping conditions (b).
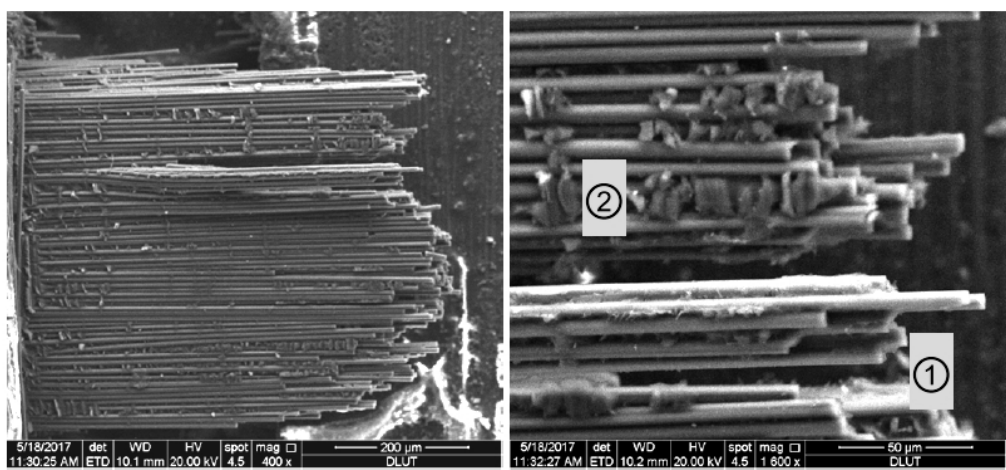

Fig. 5. SEM micrographs of the specimen's fracture: (1) fiber fracture; (2) fiber/matrix debonding.

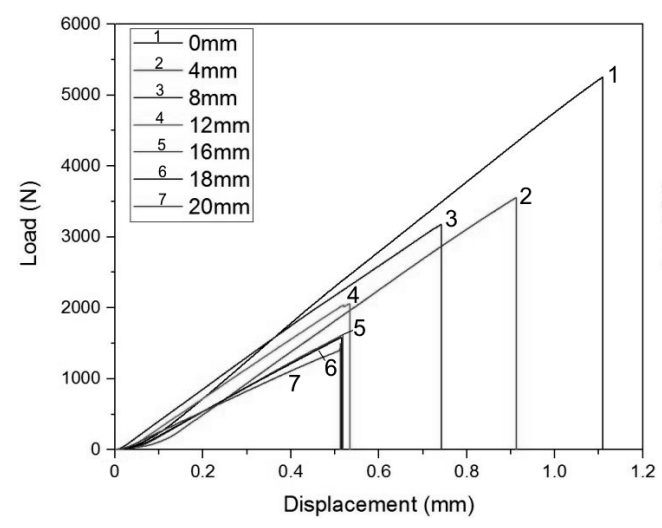

Fig. 6

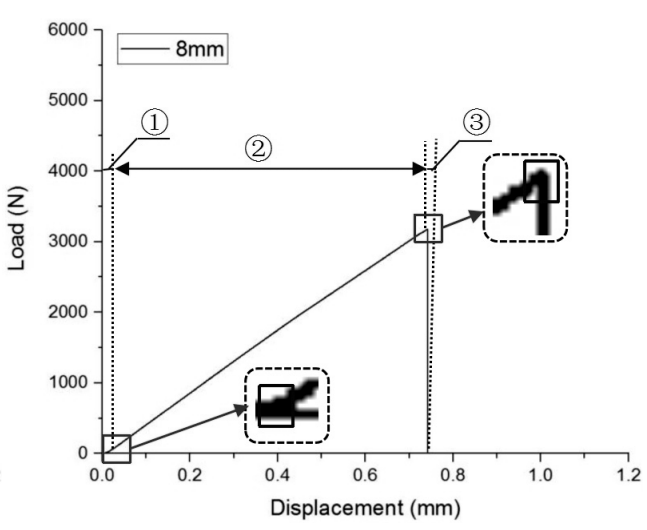

Fig. 7

Fig. 6. Load-displacement curve of specimens with pre-crack length to width ratios $(a / w)$ of $0,0.1$, $0.2,0.3,0.4,0.45$, and 0.5 .

Fig. 7. Load-displacement curve of specimens with $a / w=0.2$.

4.2. Crack Effect on the Specimen Bearing Performance. Representative loaddisplacement curves for each tested specimen with different crack lengths are shown in Fig. 6. Each of these curves can be subdivided into three stages, as shown in Fig. 7 for a 
particular example of $a / w=0.2$. The first stage involves the gap elimination, the second one is the load-bearing stage, while the third one is a fast failure stage. Observing the Fig. 8 in the second stage of the force-displacement curve, the slope of the curve decreases as the crack length increases, indicating that the bearing performance of the specimen decreases as the crack length increases.

The relationships between crack length-to-width radio and the ultimate load (ultimate displacement) are integrated in Fig. 9. The comparative analysis of curves in Figs. 7 and 9 reveals that as the crack length increases (as well as $a / w$ ratio), the bearing performance of the specimen is deteriorated, and the loading displacement is reduced.

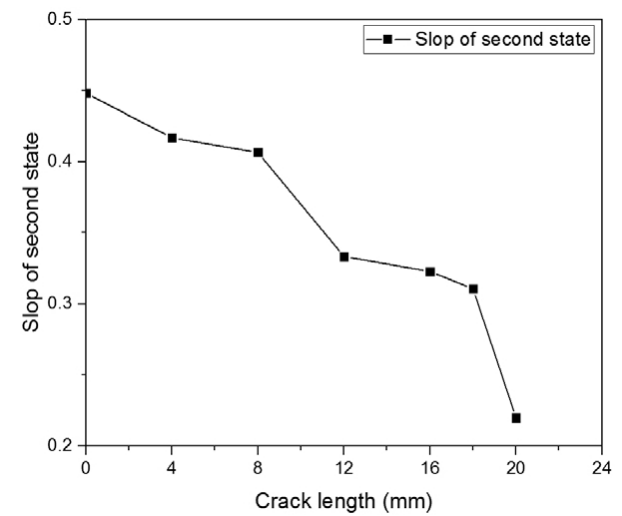

Fig. 8

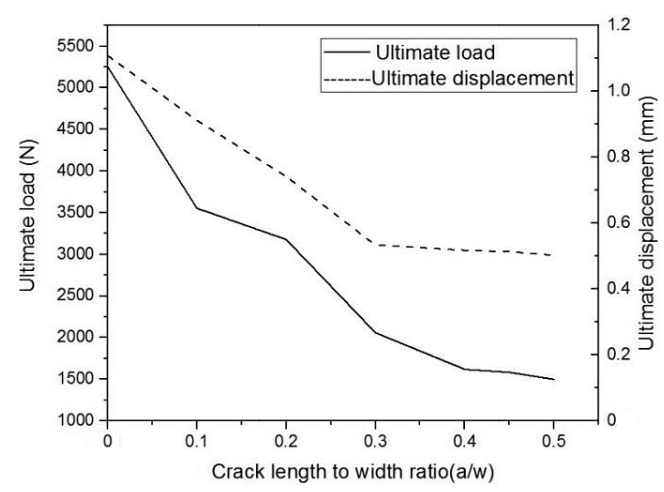

Fig. 9

Fig. 8. The slope of the second state of the load-displacement curve versus crack length.

Fig. 9. The ultimate load and displacement versus crack length-to-width ratio.

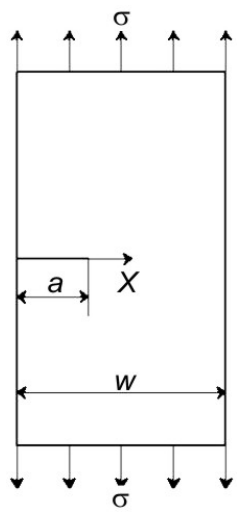

Fig. 10. Finite plate with an edge crack in tension.

4.3. Analysis and Calculation of SIF. The stress intensity factor (SIF) describes the stress field near the elastic crack tip and controls the crack tip displacement. The critical Mode I SIF for tensile stress $\sigma$ is calculated via Eq. $(15)[19,20]$ :

$$
K_{\mathrm{I}}=\sigma \sqrt{\pi a} f(a / w)
$$

where $a$ is crack length, $w$ is plate width, and $f(a / w)$ is shape factor function.

For a finite plate with an edge crack (Fig. 10), the above equation takes the following form: 


$$
K_{\mathrm{I}}=\sigma \sqrt{\pi a}\left[1.12-0.231\left(\frac{a}{w}\right)+10.55\left(\frac{a}{w}\right)^{2}-21.72\left(\frac{a}{w}\right)^{3}+30.39\left(\frac{a}{w}\right)^{4}\right] .
$$

The critical stress intensity factors of the different pre-crack length were calculated via Eq. (16) and depicted in Fig. 11.

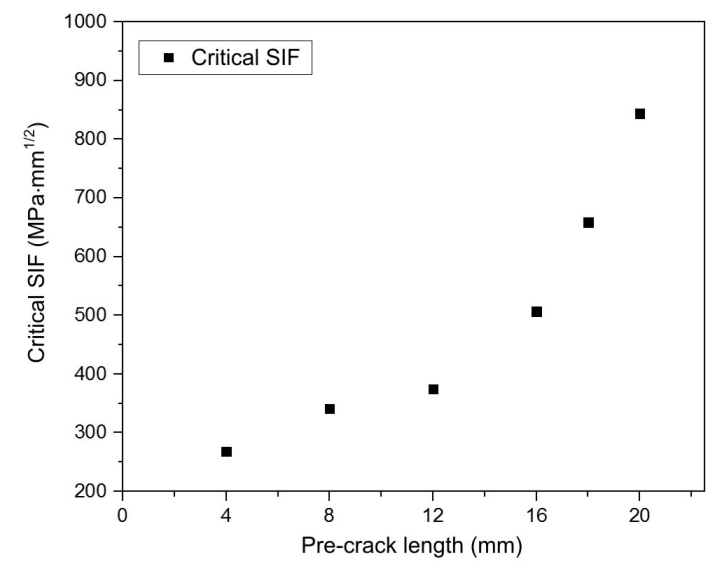

Fig. 11. The relationship between pre-crack length and critical SIF.

Conclusions. The fracture behavior and crack propagation mechanism of the unilayer composites plates are explored in this study. Based on the experimental data and numerical calculations, the following conclusions can be drawn:

1. In the crack propagation test, as a result of uni-layer plate specimens, the crack propagation process, fracture surfaces, and fracture mode could be directly observed. The warp bundles perpendicular to the crack propagation direction, the interweaving points between the weft bundles, and warp bundles are high-stress intensity zones.

2. Fracture of specimens occurred under the joint action of normal and shear stresses. In the warp and weft fiber bundle interface, because of the intersection, a wave-shaped incision was formed.

3. Particular values of the critical stress intensity factor for different crack lengths were calculated, which are considered instrumental for the residual life predictions.

Acknowledgments. This work was supported by the National Natural Science Foundation of China (Grant No. 51605076, Grant No. 51375068, and Grant No. 51475073), the National Key Basic Research Program of China (Grant No. 2014CB046504), and the Liaoning Province Natural Science Foundation of China (Grant No. 201602171).

1. N. Liu, X. F. Yao, J. D. Chen, et al., "Research on the impact damage and fracture behavior of braided composites," J. Exp. Mech., 217, No. 2, 184-190 (2002).

2. N. Blanco, D. Trias, S. T. Pinho, and P. Robinson, "Intralaminar fracture toughness characterisation of woven composite laminates. Part II: Experimental characterisation," Eng. Fract. Mech., 131, 361-370 (2014).

3. T. K. Jacobsen and B. F. Sørensen, "Mode I intra-laminar crack growth in composites modelling of R-curves from measured bridging laws," Compos. Part A-Appl. S., 32, No. 1, 1-11 (2001).

4. M. W. Czabaj and J. G. Ratcliffe, "Comparison of intralaminar and interlaminar mode-I fracture toughness of unidirectional IM7/8552 graphite/epoxy composite," in: 15th US-Japan Conference on Composite Material, NF1676Ll-15094 (2012). 
5. A. Arguelles, J. Vina, A. F. Canteli, et al., "Interlaminar crack initiation and growth rate in a carbon-fibre epoxy composite under mode-I fatigue loading," Compos. Sci. Technol., 68, No. 12, 2325-2331 (2008).

6. M. V. Donadon, B. G. Falzon, L. Iannucci, and J. M. Hodgkinson, "Intralaminar toughness characterisation of unbalanced hybrid plain weave laminates," Compos. Part A-Appl. S., 38, No. 6, 1597-1611 (2007).

7. N. Blanco, D. Trias, S. T. Pinho and P. Robinson, "Intralaminar fracture toughness characterisation of woven composite laminates. Part I: Design and analysis of a compact tension (CT) specimen," Eng. Fract. Mech., 131, 349-360 (2014).

8. A. Nasirmanesh and S. Mohammadi, "XFEM buckling analysis of cracked composite plates," Compos. Struct., 131, 333-343 (2015).

9. S. Mohammadi, XFEM Fracture Analysis of Composites, John Wiley \& Sons, Hoboken, NJ (2012).

10. A. Asadpoure and S. Mohammadi, "Developing new enrichment functions for crack simulation in orthotropic media by the extended finite element method," Int. J. Numer. Meth. Eng., 69, No. 10, 2150-2172 (2010).

11. T. Belytschko and T. Black, "Elastic crack growth in finite elements with minimal remeshing," Int. J. Fract. Mech., 45, No. 5, 601-620 (1999).

12. A. Asadpoure, S. Mohammadi, and A. Vafai, "Crack analysis in orthotropic media using the extended finite element method," Thin Wall. Struct., 44, No. 9, 1031-1038 (2006).

13. A. Asadpoure, S. Mohammadi, and A. Vafai, "Modeling crack in orthotropic media using a coupled finite element and partition of unity methods," Finite Elem. Anal. Des., 42, No. 13, 1165-1175 (2006).

14. C. Carloni and L. Nobile, "Crack initiation behaviour of orthotropic solids as predicted by the strain energy density theory," Theor. Appl. Fract. Mec., 38, No. 2, 109-119 (2002).

15. C. Carloni, A. Piva, and E. Viola, "An alternative complex variable formulation for an inclined crack in an orthotropic medium," Eng. Fract. Mech., 70, No. 15, 2033-2058 (2003).

16. A. Piva and E. Viola, "Crack propagation in an orthotropic medium," Eng. Fract. Mech., 29, No. 5, 535-548 (1988).

17. W. Y. Zhang, A Numerical Model of Woven Fabric Composite, Tsinghua University, Beijing (2002).

18. R. Seifi and N. Khoda-yari, "Experimental and numerical studies on buckling of cracked thin-plates under full and partial compression edge loading," Thin Wall. Struct., 49, No. 12, 1504-1516 (2011).

19. H. Tada, P. C. Paris, and G. R. Irwin, The Stress Analysis of Cracks Handbook, ASME Press, New York (2000).

20. T. L. Anderson, Fracture Mechanics Fundamentals and Applications, CRC Press, Boca Raton (1991). 